\title{
A New Stationary Phase with Improved Ligand Morphology Prepared by Polymerization of Styrene upon Initiator-attached Lichorsorb Silica Particles
}

\author{
Dong Guk Hwang, Shabi Abbas Zaidi, and Won Jo Cheong-
Department of Chemistry, Nano Fine Center, and Institute of Basic Research. Inha Chiversit, Incheon 402-751. Korea
"E-mail: wicheongainha.ackr
Received August 22, 2009, Accepted October 25, 2009

\begin{abstract}
Key Words: Initiator attached Lichrosorb silica. RAFT polymerization. Polystyrene bound silica. Improved ligand morphology
\end{abstract}

There have been extensive studies in controlled polymerization of organic monomers on porous or nonporous inorganic surface by making use of a surface attached initiator. The resultant organic-inorganic combined polymer can be used as stationary phases in chromatography ${ }^{1 \cdot 16}$ Various silica materials including porous silica particles. ${ }^{1.4 .5-11-13.14-16}$ silica beads. $2 \leqslant 10$. silica monolith ${ }^{9}$ and silica capillary ${ }^{3.8}$ have been used for such purposes. Atom transfer radical polymerization (ATRP) was used in the majority ${ }^{1-16.16}$ of those studies while reversible addition-fragmentation chain transfer (RAFT) polymerization was used much less frequently. ${ }^{1-13.14-15}$ Diazo type initiators were also used to be attached to silica. ${ }^{13-14}$ Coupling of RAFT polymerization to a diazo type initiator was tried. too. ${ }^{1+}$ In a particular study an initiator was attached not to silica but to porous spherical organic monolith particles, and a poly mer layer with ion exchange capability was added by ATRP. In addition, molecule imprinted polymers (MIP) were formed on the silica surface in some studies. ${ }^{15.13,15}$

In ATRP. a catalytic mixture of cuprous/cupric halides and an amine base is used to activate polymerization on the silica surface with a halogen-containing initiator. The details of principles and applications of ATRP have been well summarized in some review articles. ${ }^{18-1 \%}$ It is known that bnushes of well determined polymer of high MW with low dispersity are formed on silica surface in ATRP. In some of the ATRP studies the resultant stationary phases were reported to show better separation selectivities than conventional $\mathrm{C} 18$ phases. For example, a pheny lalanine based polymer-silica composite showed enhanced selectivities in separation of polynuclear aromatic hydrocar-

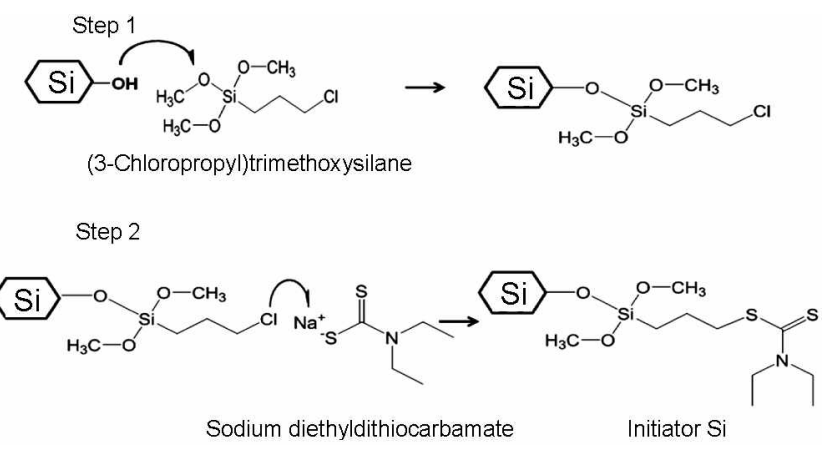

Scheme 1. The two step procedure of synthesis of the initiator attached silica. bons. ${ }^{16}$ However the reported separation efficiencies (numbers of theoretical plates) of the stationary phases were yet considerably lower than those of conmercial C 18 phases.

Attachement of a diazo type initiator onto the silica surface is not favored. for off-surface bulk solution polymerization overwhelms the surface polymerization when the initiator is activated.

In RAFT polymerization. the initiator is made by attaching a halogen containing ligand to silica surface followed by reaction with sodium diethyldithiocarbantate. Readers are referred to some review articles ${ }^{y \wedge 1}$ for principles and applications of RAFT polymerization. According to the RAFT polymerization mechanism. ${ }^{2-21}$ the polymer chain is introduced and grown in the $\mathrm{C}-\mathrm{S}$ bond located between the silyl propyl chain moiety and the diethylditlio carbanate moiety of the initiator silica shown in Scheme 1. The necessary initial radicals are generated by thermal self initiation of styrene. ${ }^{21}$ No additional catalyst is required for polymerization. Rather disappointing separation efficiencies were. however, reported with the resultant stationary phases so far ${ }^{11-12.1+-15}$ in comparison with the conventional Ci8 phases.

In our previous study, we reported an initiator silica prepared by reacting silica particles with 4-(chloromethyl) phenylisocyanate followed by sodium diethyl dithiocarbarnate and the polystyrene bound silica stationany plase thereof showing better separation efficiency and shorter retention times in contparison with the conventional $\mathrm{C} 18$ phase. ${ }^{22}$ The thickness of polystyrene layer was not uniform over the silica surface and some lumps of aggregated polystyrene were formed to cause reduction of accessible pores and surface area. Despite the good separation efficiency of such phase. we concluded that it would be better to form a uniform and thin polystyrene layer over the whole porous surface (outer and inner) of silica to obtain further improved separation efficiency.

In this study porous silica particles were reacted with 3-chloropropyltrimethoxysilane followed by sodium diethyl dithiocarbannate. The porous initiator silica particles were then allowed to undergo radical poly merization with styrene to give a new stationary phase. This stationary phase was packed in a glasslined stainless steel microcolumn to examine the chromatographic performance. We were able to attain the organic-inorganic combined polymer stationary phase with improved ligand morphology to show extended retention times and generally better separation performance than that of the conventional CI8 phase. 


\section{Experimental}

Materials. Sty'rene. 3-chloropropy'l trimethoxy silane. sodium diethyl dithiocarbamate. anhydrous tetrahydrofuran (THF). anhydrous toluene, anhy drous p-xylene, 1, 1,1,3.3.3 hexamethyldisilazane (HMDS), trimethylchlorosilane (TMCS). chlorooctadecyldimethylsilane were purchased from Sigma-Aldrich (St. Louis, MO, USA). HPLC grade methanol, acetonitrile. water were purchased from Mallinckrodt Baker (Phillipsburg. NJ USA). All the reagents were used as received. Screen frits (1.6 mm diameter, $0.08 \mathrm{~mm}$ thickness) were purchased from Valco (Houston. TX. USA). The Lichrosorb Si 100 (5 $\mu \mathrm{m} .100$ A. $300 \mathrm{~m}^{3} / \mathrm{g}$ ) silica particles were purchased from MERCK (Danmstadt. Germany). Pieces of glass lined stainless steel tubing ( $30 \mathrm{~cm}, 0.5 \mathrm{~mm}$ I.D. $1.6 \mathrm{~mm}$ O.D.) were purchased from Alltech (Deerfield. IL. USA).

Synthesis of the initiator silica. The initiator attached silica was prepared by reacting the silica particles with 3-chloropropyl trimethoxysilane followed by sodium diethyl dithiocarbamate. In the first step. the $500 \mathrm{mg}$ silica particles were dried at $120^{\circ} \mathrm{C}$ for 4 h or more, and dispersed in a mixture of $2 \mathrm{~mL} \mathrm{3-chloro-}$ propyl trimethoxy'silane and $8 \mathrm{~mL}$ anhydrous toluene. Reaction was carried out under reflux at $110^{\circ} \mathrm{C}$ for $24 \mathrm{~h}$. After completion the product was thoroughly washed with toluene followed by acetone. filtered, and air dried.

In the second step. $200 \mathrm{mg}$ sodium diethyl dithiocarbamate was dissolved in $10 \mathrm{mLTHF}$, and the solution was purged by nitrogen to remove oxygen, then the initiator-attached silica particles were suspended in the solution and reacted at $55^{\circ} \mathrm{C}$ for $10 \mathrm{~h}$. The product was thoroughly washed with THF and acetone in sequence. filtered. dried and stored in vacuum desiccators at room temperature.

The preparation scheme of the initiator attached silica is shown in Scheme 1.

Polymerization of styrene upon the initiator attached silica. Styrene $2.5 \mathrm{~mL}$ was dissolved in $4 \mathrm{~mL} p$-xylene, and the solution was purged by nitrogen, then the $100 \mathrm{mg}$ initiator silica particles were suspended by stirring and radical polymerization was carried out under reflux at $110^{\circ} \mathrm{C}$ for $40 \mathrm{~h}$. The polysty rene combined silica particles were thoroughly washed with toluene. methanol and acetone in sequence and dried at $60^{\circ} \mathrm{C}$ for $10 \mathrm{~h}$.

C18 modification and end-capping of bare silica. An exact amount of $200 \mathrm{mg}$ silica was modified with $0.12 \mathrm{~g}$ chlorodimethyl octadecylsilane dissolved in $5.0 \mathrm{~mL}$ anhydrous toluene in a small round bottom flask by stiring at $110^{\circ} \mathrm{C}$ for $10 \mathrm{~h}$. then encapped by adding the mixture of $105 \mu \mathrm{L}$ HMDS and $35 \mu \mathrm{L}$ TMCS slowly with a dropping funnel at $70^{\circ} \mathrm{C}$ for 6 h or more. The final product was filtered with an Alltech (Deerfield. IL. USA) PTFE $1 \mu \mathrm{m}$ membrane filter and washed with toluene, methanol and acetone in sequence and dried at $60^{\circ} \mathrm{C}$ for $10 \mathrm{~h}$.

$\boldsymbol{\mu L C}$. A Shimadzu (Tokyo. Japan) I0AD pump. a Shimadzu DGU-14A membrane degasser, a Valco (Houston. TX. USA) CI+W.05 injector with a $50 \mathrm{~nL}$ injection loop. a Jasco (Tokyo. Japan) UV-2075 UV/Vis capillary window detector, and the home-made $0.5 \mathrm{~mm}$ I.D. glass-lined micro column were assembled to construct the $\mu \mathrm{LC}$ system. A PC system with the software Multichro 2000 from Youlin-Gisul (Sungnam. Korea) was used to acquire and process the chromatographic data. The micro columns were packed according to the procedure reported before ${ }^{2-2 \cdot 24}$ with some modifications. Thus, a frit (commercial screen frit) was placed in the $1 / 16$ inch outlet of a $1 / 16-1 / 32$ reducing union a piece of $30 \mathrm{~cm}$ glass lined stainless steel tubing was fitted to the outlet. and the tubing was connected to the packer. The $100 \mathrm{mg}$ particles were suspended in $1.2 \mathrm{~mL}$ methanol. and sonicated for $10 \mathrm{~min}$. The pressure of the slumy packer was instantly raised to $14.000 \mathrm{psi}$ for $4 \mathrm{~min}$, adjusted to 10,000 psi for the next $15 \mathrm{~min}$ followed by setting at $8.000 \mathrm{psi}$ for 40 min. Finally the pressure was raised again to 14,000 psi for 4 min. The initial high pressure step ( $14,000 \mathrm{psi}$ ) was the most crucial step to set up a good basic packing structure, and the next two steps were necessary to condition (settle down) the packing. and the final step was required to eliminate tiny crevices in the packing. Then the compressor was turned off to release the pressure on standing for a while. The colunn was then connected to the injector through a union with a piece of $5 \mathrm{~cm}$ stainless steel tubing ( $100 \mu \mathrm{m}$ I.D., 1.6 nun O.D.) and a frit. The $1 / 32$ inch outlet of the other column end was connected to the capillary window detector by installing a $1 / 32$ inch graphite ferrule and a connecting capillary ( $50 \mu \mathrm{m}$ I.D., $365 \mu \mathrm{m}$ O.D.). A piece of short Teflon tubing was used to connect the two capillary ends.

A test mix composed of phenol, acetophenone, 4-methyl-2nitroaniline, benzene, and toluene was prepared in acetonitrile and stored in a refrigerator at $4^{\circ} \mathrm{C}$ before use. The stock sample solution was composed of $1.2 \mu \mathrm{L}$ phenol, $2 \mu \mathrm{L}$ acetophenone. $1.5 \mathrm{mg}+$-methyl-2-nitroaniline, $6 \mu \mathrm{L}$ benzene, and $+\mu \mathrm{L}$ toluene in $1 \mathrm{~mL}$ solvent. The stock sample was diluted enough with the mobile phase as long as sufficient signal sensitivity was secured to avoid sample overloading.

\section{Results and Discussion}

Architecture of stationary phases. The SEM photos of the polystyrene bound initiator silica and the C 18 modified silica in different view scales are compared in Figure 1. and the elemental analysis results of the initiator silica, the polysty rene bound silica, and the C18 modified (and end-capped) silica of this study are summarized in comparison with those of the previous study in Table 1

(a)

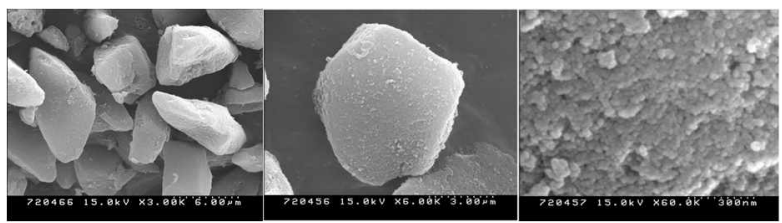

(b)

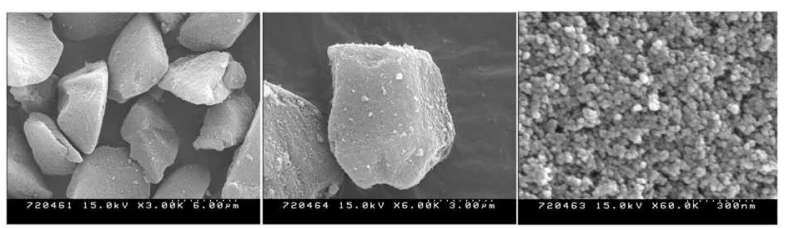

Figure 1. Comparison of the SEM images of (a) the polystyrene bound silica and (b) the $\mathrm{C} 18$ bound silica. From the left, the view of multiple particles, the view of single particle, and the expanded view of the surface of single particle are shown. 
Table 1. Comparison of the EA results among the initiator silica, the polystyrene bound initiator silica, the C1 8 bound silica of this study, and the initiator silica and the polystyrene bound initiator silica of the previous study

\begin{tabular}{lccccc}
\hline Element & Initiator silica & $\begin{array}{c}\text { Polystyrene bound } \\
\text { initiator silica }\end{array}$ & C18 bound silica & $\begin{array}{c}\text { Initiator silica of } \\
\text { previous study }\end{array}$ & $\begin{array}{c}\text { Polystyrene bound } \\
\text { intiator silica of } \\
\text { previous study }\end{array}$ \\
\hline Carbon & $6.51 \%$ & $19.96 \%$ & $10.63 \%$ & $4.80 \%$ & $16.70 \%$ \\
Hydrogen & $0.90 \%$ & $1.98 \%$ & $1.96 \%$ & $0.60 \%$ & $1.40 \%$ \\
\hline
\end{tabular}

Table 2. Comparison of the values of number of theoretical plates (averages and standard deviations) based on 5 batches of micro columns ( $0.5 \times$ $300 \mathrm{~mm}$ ) among the polystyrene bound initiator silica, the C1 8 bound silica of this study, and the polystyrene bound silica of the previous study ${ }^{22}$

\begin{tabular}{lccc}
\hline Solute & $\begin{array}{c}\text { Polystyrene bound silica of } \\
\text { this study }\end{array}$ & Cl8 bound silica & $\begin{array}{c}\text { Polystyrene bound silica of the } \\
\text { previous study }\end{array}$ \\
\hline phenol & $26000 \pm 500$ & $21200 \pm 600$ & $17700 \pm 500$ \\
acetophenone & $24100 \pm 400$ & $20300 \pm 400$ & $16800 \pm 700$ \\
4-methyl-2-nitroaniline & $22000 \pm 900$ & $22100 \pm 800$ & $18700 \pm 800$ \\
benzene & $21800 \pm 400$ & $20800 \pm 500$ & $19100 \pm 600$ \\
toluene & $19500 \pm 800$ & $21100 \pm 600$ & $21600 \pm 500$ \\
\hline
\end{tabular}
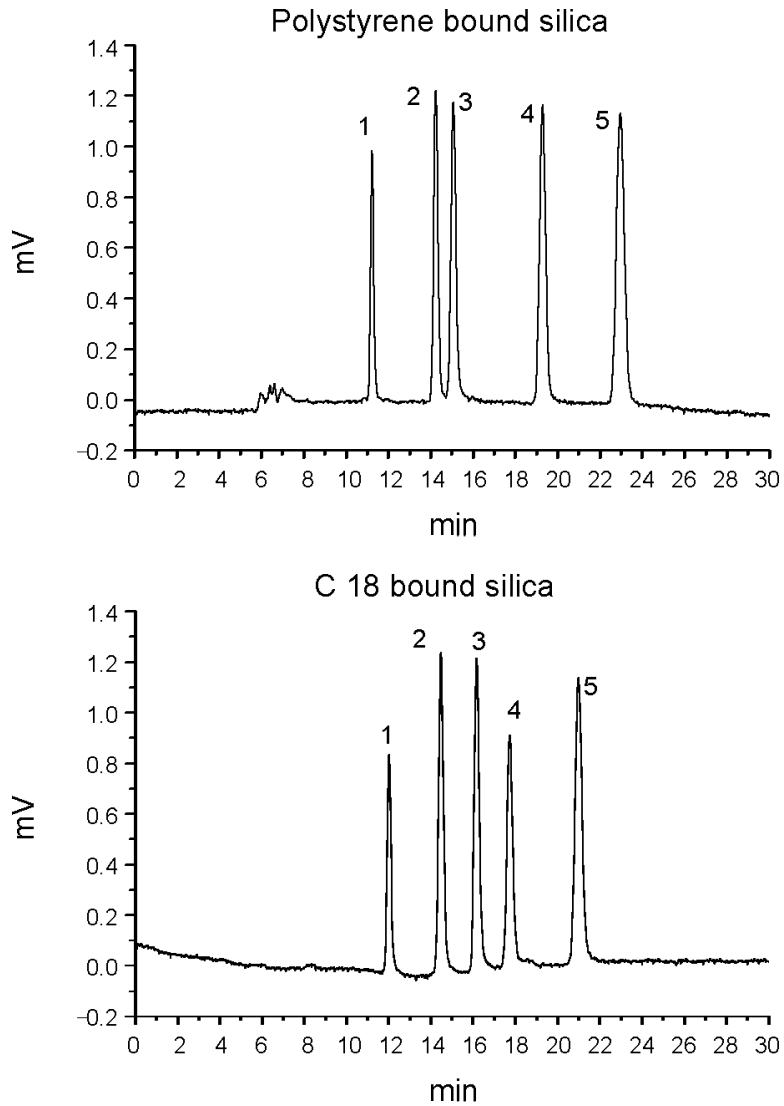

Figure 2. Comparison of the chromatograms obtained by the polvstyrene combined initiator silica and the C 18 bound (and end-capped) silica in $70 / 30(\mathrm{v} / \mathrm{v})$ acetonitrile/water with $0.1 \%$ TFA at a flow rate of $7 \mu \mathrm{L} / 111 \mathrm{n} 1$ at $214 \mathrm{~cm}$. 1: phenol, 2; acetophenone, 3: 4-methyl-2nitroaniline, 4 ; benzene, 5 ; toluene.

In our previous study ${ }^{27}$ where a different initiator was attached to silica. the resultant stationary phase had a polysty rene layer of irregular thickness. and some lumps of aggregated polysty rene were formed probably' owing to heterogeneous formation of polystyrene.
Rather homogeneous polymerization was achieved with the new initiator silica in this study as shown in Figure 1. There is no identifiable difference in the SEM images of single or multiple particles between the polysty rene bound silica (Figure la) and the $\mathrm{C} 18$ bound silica (Figure $\mathrm{lb}$ ). There is, however, certainly a clear difference in the expanded SEM images of the surface of a single particle. The surface of the $\mathrm{Cl} 8$ bound silica was very porous and similar to that of bare silica while the surface of the polysty rene bound silica was much less porous owing to formation of a rather uniform thin layer of polystyrene. There were no large poly'styrene lumps on the surface of the polystyrene bound silica.

Comparison of separation performance among the polystyrene combined Lichrosont silica, the C18 modified Lichrosorb silica of this study, and the polystyrene combined Adsortosphere silica of the previous study. Two identical microcolums $(300 \times 0.5 \mathrm{~nm})$ were packed with the polysty rene combined initiator silica particles and the C 18 modified and end-capped silica particles. respectively, to compare their chromatograplic performances. The same chromatographic conditions were used for both colunins. The chromatographic results were compared in Figure 2. The data of number of theoretical plates $(\mathrm{N})$ of the poly'styrene bound initiator silica. the $\mathrm{C} 18$ bound silica of this study, and the polystyrene bound silica of the previous study ${ }^{22}$ were compared in Table 2.

As slown in Figure 2 and Table 2. the separation performance of the polystyrene bound silica was found generally better than that of the $\mathrm{C} 18$ bound silica. Furthermore. congestion of peaks observed in the previous study ${ }^{22}$ owing to reduction of solute retention times was avoided in this study as expected from the improved morphology of the present polystyrene bound silica. Of course the separation efficiency of the poly sty rene bound silica of this study was considerably better than that of the previous study ${ }^{22}$ as shown in Table 2. Such improvenent seems to be enabled by the proper choice of attached initiator. The surface attached initiator of this study was believed to induce proper polymerization kinetics and thermodynamics preventing heterogeneous growth of large polystyrene lunups. Details 
of such theory may be very complicated and subject to future study.

It should be noted that the solute retention times obtained with the polystyrene bound silica were comparable to those obtained with the C 18 bound silica (Figure 2) although the carbon load of the former was almost as twice as that of the latter (Table 1). This means that the accessible surface area/mass ratio of the poly sty rene ligand should be much lower than that of the $\mathrm{C} 18$ ligand. It can be explained by the assumption that a large portion of the inner pores of the bare silica are filled (clogged) when polymerization takes place while such effect is minimal when $\mathrm{C} 18$ ligands are attached. Actually, $50 \%$ reduction of the pores was reported in preparation of polymer bound silica in the literature. ${ }^{14}$

Thus. $\mathrm{N}_{2}$ adsorption/desorption experiments were carried out for our phases. The pore volume of the bare silica. $\mathrm{C} 18$ bound silica and polysty rene bound silica were found to be $0.64 .0 .54,0.32 \mathrm{~cm}^{3} / \mathrm{g}$, the BJH adsorption surface area. 343 . $237.168 \mathrm{~m}^{2} / \mathrm{g}$. and the average adsorption pore size. 133.133. $131 \mathrm{~A}^{\circ}$. respectively. It can be noted that the pore volume and surface area were reduced a lot by formation of poly sty rene.

It was also mentioned that the polymer MW of the outer surface was higher than that of the inner pores since transfer of monomer into the inner pores was much slower. ${ }^{14}$ Such a situation was found to be prevailing in our phase. too. The initiator density was calculated to be $6.0 \times 10^{-4} \mathrm{~mol} / \mathrm{g}$ (or $2.0 \mu \mathrm{mol} / \mathrm{m}^{2}$ ) based on the elemental analy sis data (Table 1). and the amount of styrene monomer in the polysty rene bound silica was estimated to be $1.35 \times 10^{-7} \mathrm{~mol} / \mathrm{g}$. Thus ca 2.3 sty rene molecules were attached to an initiator molecule on average. which seems to be unreasonable. We believe that a large portion of the initiator molecules in deep and/or small pores were not actually used for formation of polystyrene oligomers owing to either clogging of the upper level pores or poor accessibility and that the initiator molecules on the outer surface were subject to accepting more styrene molecules than the above average mumber. It is supported by the observation that the pore size of the polysty rene bound silica was similar to that of the bare silica while the pore volume of the polysty rene bound silica was about half of that of the bare silica. Such a phenomenon can occur only if the inner pores are clogged when poly merization takes place.

Thus the particle of our phase can be regarded to have a pseudo core-shell structure where the deep core is closed. the shallow pores are partially filled, and the outer surface is smoothly covered with polystyrene oligomers with relatively high molecular weights.

\section{Conclusion}

A new stationary phase has been prepared by synthesizing new initiator silica and forming polystyrene on the silica surface by reversible addition-fragmentation chain transfer polymerization. A polystyrene bound silica phase with improved morphology was obtained and its separation efficiency was better than that of the polystyrene bound silica phase of the previous study The separation efficiency of the new stationary phase was generally better than that of the $\mathrm{C} 18$ modified silica phase.

Aclonowledgments. This work was supported by the 2009 Inha University Fund.

\section{References}

1. Rahman, M. M:; Czaun, M.; Takafuji, M:- Ihara, H. Chem. Eur. J. 2008, 1t, 1312-1321

2. Nagase, K, Kobayashi, T.: Kihnchi, A.: Akivama, Y.: Annaka, M: Kanazawa, H.; Okano, T. Langmuir 2008, 24, 10981-10987.

3. Idota, N.; Kikuchi, A.; Kobavashi, T.; Akivama, Y : Sakai, K.; Okano, T. Lomgnuir 2006, 22, 425-430.

4. Coad B. R.; Steels, B. M.; Kizhakkedathu, J. N.; Brooks, D. E.; Haynes, C. A. Biotechnol Bioengineer: 2007, 97, 574-587.

5. Nagase, K: Kobayashi, T.; Kiklkhi, A : Akiyama, Y.; Kanazawa, H.; Okano, T. Longntuir 2008, 24,5l 1-517.

6. Mallik, A. K.; Rahman, M. M.; Czaun, M:- Takafuji, M.; Ihara, H. J. Chromatogr. \& 2008, 1187, 119-127.

7. Hemstrom, P.; Szumski, M.: Irgum, K. Anal Chem 2006, 78 , 7098-7103

8. Miller, M. D.; Baker, G. L.; Bruening, M. L. J. Chrontatogr. A $2004,1044,323-330$.

9. Yoshikawa. C.: Goto. A.: Tsujii, Y.: Ishizuka. N.: Nakanishi. K.: Fukuda, T. J. Polm. Sci. Pt.4. Polm Chem. 2007, 45, 4795-4803.

10. Nagase, K.; Kobayashi, T.; Kikuchi, A.; Akivama, Y.; Kanazawa, H.; Okano, T. Biomacromolecules 2008, $9,1340-1347$.

11. Derollet, D; Thuc, C. N. H. J. Appl Polm Sci 2008, 109, 21132127

12. Fairhurst, R. E; Chassaing, C.; Vent, R. F.; Mayes, A. G. Biosensors and Bioelectronics 2004, 20, 1098-1105.

13. Sulitzky, C.; Ruckert, B.: Hall, A. J.; Lanza, F.; Unger, K.: Sellergren, B. Lacromolecules 2002, 35, 79-91

14. Roohi. F.; Titirici, M. M. New J. Chent 2008,32, 1409-1414.

15. Su, S.; Zhang, M, Li, B.; Zhang, H,; Dong, X. Talanta 2008, 76 , 1141-1146.

16. Czaun, M.; Rahman, M. M: Takafuji, M.; Ihara, H. Pohmer 2008. $49.5410-5416$

17. Unsal, E; Elmas, B.; Caglayan, B.; Tuncel, M.; Patir, S.; Tuncel, A. Anal. Chent 2006, 78, 5868-5875

18. Coessens, V.; Pintaner, T.: Matyjaszewski, K. Prog. Polmm. Sci 2001, 26, 337-377

19. Qiu, K, Li, P. Chinese J. Polm Sci. 2004, 22, 99-110

20. Favier, A.; Charreyre, M. Hacromol. Rapid Comm. 2006, 27, $653-692$.

21. Perrier, S.: Takolpuckdee, P. J. Pohm Sci. Pt. A: Polym. Chem. $2005,43,5347-5393$.

22. Kim, S. S.; Cheong, W. T. Bull. Korean Chem. Soc. 2009, 30, 722725 .

23. Kim, S. S.: Cheong, W. I. Bull. Korean Chem. Soc. 2008, 29, $1627-1629$

24. Cheong, W. . .; Go, J. H.; Baik, Y. S.; Kim, S. S.; Nagarajan, E. R.; Selvapalam, N.; Ko, Y. H.; Kim, K. Bull. Korean Chem. Soc. 2008, 29, 1941-1945. 